\title{
La Educación inclusiva en la realidad educativa chilena
}

\author{
Karina Raquel Vasquez ${ }^{1}$, Carlos Ángel Mancilla Guzmán², Victor Daniel Muñoz Cárdenas ${ }^{3}$ \\ y Jesse Andrés Obreque Jara ${ }^{4}$ \\ ${ }^{1234}$ Universidad Adventista de Chile
}

Recibido: 16 de noviembre de 2016

Aceptado: 16 de enero de 2017

\section{Resumen}

La presente revisión teórica pretende dar a conocer la realidad chilena actual sobre el cambio curricular, que apunta a una visión inclusiva de la educación. Este estudio se realizó por medio de una revisión bibliográfica del contexto en la cual se encuentra la educación chilena y su relación con las Necesidades Educativas Especiales, a través de un análisis educativo de la legislación y el curriculum educativo, destacando aspectos fundamentales sobre una estructura metodológica diversificada. Se concluye con una visualización sobre el cambio en la legislación y los factores que son relevantes para una educación diversificada, inclusiva y para todos los estudiantes.

Palabras clave: Visión inclusiva de la educación, necesidades educativas especiales, eurriculum educativo, educación diversificada.

\begin{abstract}
The present theoretical revision aims to present the current Chilean reality about curricular change, which points to an inclusive vision of education. This study was carried out by means of a bibliographical review of the context in which Chilean education and its relation with Special Educational Needs are found, through an educational analysis of the legislation and the educational curriculum, highlighting fundamental aspects
\end{abstract}

\footnotetext{
${ }^{1}$ Correspondencia al autor

E-mail: karinavasquez@unach.cl
} 
about a methodological structure Diversifies. It concludes with a view on the change in legislation and factors that are relevant to a diversified, inclusive education for all students.

Keywords: Inclusive vision of education, Special Educational Needs, Educational Curriculum, Diversified education.

\section{Introducción}

¿Qué es educar?, como respuesta se plantean ideas de cambio en estas últimas décadas sobre la educación y el desarrollo de los niños, que son parte de las buenas prácticas docentes. Rodríguez (2008), menciona, que en las últimas décadas la educación presenta presiones y un rol social, así como un incremento comunicacional con una renovación acelerada debido a las exigencias de calidad del sistema imperante. Por otro lado, Álvarez, Porta y Sarasa (2011) se refieren que el profesor debe poner énfasis hacia el clima escolar, donde cobra importancia el tipo de lenguaje y feedback que se tiene con el alumno, otorgando importancia al tiempo y atención generada hacia situaciones particulares del estudiante.

La percepción de los estudiantes en el clima escolar según Guerra y Cols (2012), demostraron en un estudio realizado con alumnos de enseñanza media, que los estudiantes presentan dificultades para conectar sus necesidades educativas con el resto de sus compañeros y comunidad escolar. Por otro lado, se observó que los estudiantes interaccionan de buena forma entre su propio género y el tipo de establecimiento, lo que implica una generación de confianza cuando se sienten acogidos en un grupo, disminuyendo las barreras en la educación y aumentando el desarrollo cognitivo.

Piaget, menciona que la forma en que los niños se desarrollan cognitiva y moralmente son un aspecto a considerar para determinar sus necesidades educativas, por lo tanto, sería interesante realizar evaluaciones en los diferentes establecimientos educacionales, con el objetivo de identificar si existe igual desarrollo moral en niños de las mismas edades, pero con diferente nivel sociocultural, como una estrategia para proponer innovaciones a nivel curricular, que fomente y establezca tiempos y horas lectivas para la formación del desarrollo del juicio moral (Fuentes y Cols, 2012). En este sentido, un estudio realizado por la Universidad Católica del Maule, Chile, evidencia que los niños no son seres pasivos, más bien todo lo contrario, tienen respuestas frente a 
estímulos externos, realizando acomodos para adaptarse a una nueva situación y donde el desarrollo de la inteligencia está en las respuestas adaptativas, además establece que la moral infantil permitirá entender la del hombre, postulando entonces que, para formar hombres, es útil aprender a conocer las leyes de esta formación. (Fuentes y Cols, 2012).

Maturana (2001), menciona que la educación de un joven ocurre todo el tiempo, ya que es un proceso en el cual un sujeto convive con otro y al relacionarse entre ellos se transforma espontáneamente, de esta manera, su forma de vivir se hace progresiva, según en el espacio de convivencia.

Blanco (2006) plantea que la mayoría de los países se han preocupado de adoptar políticas públicas, partiendo de una Declaración de Educación para Todos, sin embargo excluyen a innumerables estudiantes del sistema educativo. Por otra parte, Rosa Blanco plantea que se ha avanzado en un acceso a la educación universal para todos, pero con problemas de equidad y calidad en el tipo conocimiento entregado.

Desde el concepto de acceso universal nace el objetivo central del presente trabajo, que es identificar los aspectos del cambio en el paradigma de la educación, donde la importancia de la inclusión educativa convierten al modelo tradicional, en un modelo de acceso universal de todas y todos los estudiantes, donde se valoran las individualidades y oportunidades de aprender. Así como, la determinación de las Necesidades Educativas y las Necesidades Educativas Especiales, enfrentadas a un modelo global de la enseñanza.

La metodología utilizada responde a un análisis discursivo de temáticas implicadas en la educación, el cambio curricular que demanda actualmente las políticas educativas en inclusión, estudios sobre la problemática social, clima escolar, y desarrollo del estudiante en la escuela.

\section{Modelo educativo chileno}

El modelo educativo chileno parte desde el tiempo histórico de la Colonia, donde se dieron instancias en las que se impartió un determinado conocimiento, que no eran instalaciones pedagógicas conscientes, puesto que el conocimiento solo prestaba una utilizada operativa básica (Educarchile, 2005a). Por otro lado, existe un pensamiento adoptado, proveniente de un modelo 
educativo francés, incorporado como modelo pedagógico academicista o tradicional instalado a partir del siglo XIX, (Educarchile, 2011).

En cuanto a la planificación presente en este modelo educativo, se puede mencionar que es una enumeración de conceptos, donde los estudiantes deberán aprender en un determinado lapso de tiempo, que no considera la importancia y los cuestionamientos propios del sujeto, por lo tanto, se transforma en una acumulación sucesiva de aprendizajes.

Entonces de qué forma se podrá mejorar la calidad de la educación en Chile, ya que no se puede mirar únicamente la planificación y los resultados de las mediciones internacionales o nacionales del logro académico de los estudiantes, lo que implica prestar atención a temas más complejos que forman parte de la educación, como el desarrollo de capacidades para la convivencia y la tolerancia, el respeto al otro, la creatividad, la inserción en el mercado laboral, el ejercicio de la ciudadanía y la democracia. (Educarchile, 2005a).

Freire (1972) entre sus postulados plantea que la educación verdadera es praxis, reflexión y acción del hombre sobre el mundo para transformarlo. En este sentido, un estudio de Educarchile (2005b) sobre las prácticas educativas indica que:

El sistema educativo chileno necesita generar mecanismos para eliminar prácticas discriminatorias en el acceso a las escuelas. En teoría, el modelo permite que los padres elijan el establecimiento en el que matriculan a sus hijos. No obstante, existe evidencia de prácticas de selección de alumnos por parte de los establecimientos de enseñanza (estatus socioeconómico o pertenencia étnica de los estudiantes, por ejemplo) que llevan a reproducir desigualdades sociales y perennizan modelos de trato homogéneo. (párr. 9).

\section{El currículo}

En la actualidad el concepto de currículum es muy utilizado, por lo tanto es primordial analizar, definir y lograr describir si existen otros tipos de currículum.

Según Cox (2001) el currículum se asocia a los planes y programas o al conjunto de los contenidos, que son organizados de forma secuencial, con un sentido amplio por el sistema educacional, adoptando características organizadoras, donde el cambio marcado y rápido de la cultura presiona a estar actualizando y modificando los propósitos formativos, así como presta la función de organizar la trayectoria de alumnos y alumnas en el periodos de tiempo escolarizado. 
Contextualizando y retrocediendo tres décadas atrás, el cambio que se ha generado hasta ahora, es que durante la década de los ochenta estuvo inmerso por diferentes cambios sociales que influyeron en algunos aspectos la educación. Gajardo y Andraca (1988) y Espínola (1990) citado por Cox (2001) enfatizan que las medidas flexibilizadoras y descentralizadoras del currículum, en un contexto de políticas, no se guiaban por el criterio de la equidad, en consecuencia, tuvieron efectos negativos sobre la educación de los más pobres: menos horas en los planes de estudio y menos contenidos.

Acercándonos diez años más a la actualidad, el currículum se adapta a las necesidades del aprendizaje. El cambio curricular iniciado a fines de la década de 1990 responde a la necesidad del sistema escolar de adaptar "los qué" y "para qué" de las oportunidades de aprendizaje que ofrece a las mayorías.

Entre estos cambios de aprendizaje se encuentra la Ley Loce, "el primer cambio que establece la reforma curricular que comprende a la dimensión de control y regulación del currículum en el sistema escolar. Este es heredado del régimen militar y su orientación es descentralizadora" (Cox, 2001, p. 219).

Posner (1998), analiza los diferentes tipos de currículum, con características diferentes que describen una realidad educativa: El currículo Oficial: "Se describe de manera documental, a través de planes y programas, materiales didácticos, guías y los objetivos del sistema”. (p.9) Currículo Real, Vivido u Operacional: "Puesto en práctica el currículo oficial que incluye las adecuaciones del contexto del aula" (p.9). Currículo Oculto: "Normas institucionales y valores no reconocidos abiertamente por profesores y funcionarios escolares; su profundidad e impacto a veces llegan a resultar mayores que los del currículo oficial” (p.10). Currículo Nulo: Conjunto de contenidos, aprendizajes y habilidades que no están presentes en los currículos diseñados a planificados, pero constituyen una de las demandas de los alumnos o de la sociedad. Aquello que la escuela no enseña o atiende (p.10).

El tema sobre el currículo y su reforma se deben al cambio acelerado de la cultura que afectan tanto las habilidades y valores de la educación. Esto se traduce en aspectos de revolución de la información comunicativa globalizada, entre otros asociados al crecimiento económico y desarrollo democrático (Cox, 2011). 


\section{Ley general de educación}

La ley General de Educación (LGE), número 20.370, promulgada el 17 de agosto de 2009, menciona en su artículo $\mathrm{N}^{\circ}$ 1: " sobre derechos y deberes de los integrantes de la comunidad educativa, fijando los requisitos mínimos que deberán exigir cada uno de los niveles de educación parvularia, básica y media, velando por el cumplimiento, requisitos, y el proceso del reconocimiento oficial de los establecimientos es instituciones educacionales, con el objetivo que apunta a la equidad y calidad del servicio.

En primera instancia, la legislación chilena de acuerdo al Ministerio de Educación de Chile en la Ley mencionada, hace alusión al tipo de Educación Especial, donde se determina lo siguiente:

La Educación Especial o Diferencial es la modalidad del sistema educativo que desarrolla su acción de manera transversal en los distintos niveles, tanto en los establecimientos de educación regular como especial, proveyendo un conjunto de servicios, recursos humanos, técnicos, conocimientos especializados y ayudas para atender las necesidades educativas especiales que puedan presentar algunos alumnos de manera temporal o permanente a lo largo de su escolaridad, como consecuencia de un déficit o una dificultad específica de aprendizaje" (Art. 23, párr.1).

Por lo tanto, se introduce una necesidad de establecer preocupación por una modalidad de educación que ponga atención a las Necesidades Educativa Especiales.

\section{Decreto 170}

La Educación Especial o Diferencial, se regula través del decreto 170 en la ley no. 20.201, en el artículo 2, menciona al alumno que presenta Necesidades Educativas Especiales Transitorias y Permanentes, esto implica "a todo aquél que precisa ayudas y recursos, ya sean humanos, materiales o pedagógicos, para conducir su proceso de desarrollo y aprendizaje, y contribuir al logro de los fines de la educación” (Ley N²0.201, 2010).

En este sentido, las Necesidades Educativas Especiales permanentes, son aquellas dificultades en el desarrollo meta cognitivo en algún área del aprendizaje que estudiantes experimentan durante toda su escolaridad como secuela de una discapacidad diagnosticada por un profesional y que además demandan al sistema educacional para la provisión de apoyos y recursos extraordinarios (Ley $\mathrm{N}^{\circ}$ 20.201, 2010). Y en el caso las de carácter transitorio, son aquellas "no permanentes que requieren en algún momento de su vida escolar a consecuencia de un trastorno o 
discapacidad diagnosticada por un profesional competente y que necesitan de ayudas o apoyos extraordinarios" para acceder o progresar en el currículum por un determinado periodo de su escolarización. (Art. 2, párr.3).

\section{Decreto 83}

Como legislación adicional se aprueba criterios y orientaciones de adecuación curricular para estudiantes con NEE de educación parvularia y educación básica, basado en la diversificación de la enseñanza en el Decreto 83 del año 2015, donde se hace referencia a los "criterios y orientaciones de adecuación curricular" (art .3). Como el proceso a seguir para "favorecer el acceso al currículo nacional de los estudiantes con necesidades educativas y necesidades educativas especiales" (Ministerio de Educación de Chile, 2015a).

El Ministerio de Educación de Chile en el Decreto 83, menciona los principios que orientan la toma de decisiones para definir las adecuaciones curriculares, entre ellos están la valoración a la diversidad, inclusión educativa, calidad e igualdad de oportunidades.

\section{La problemática curricular}

Actualmente existe un sistema curricular que debe ser ajustado a las necesidades de la realidad en el aula de clases, ya que en este contexto es indispensable la adquisición de nuevos conocimientos. Es por esto, que surge la iniciativa de la construcción de una planificación diversificada que comprenda la entrega de conocimientos y herramientas que permitan aprendizajes significativos para estudiantes con distintas formas de aprender, así como lo describe un estudio realizado por Machado "Los sistemas educativos están basados en un modelo de educación homogénea, cuando hoy la sociedad globalizada se caracteriza cada vez más por su diversidad" (Educarchile, 2005b).

Como respuesta a la necesidad educativa, en la aplicación de la planificación diversificada tiene como propósito "un enfoque basado en la investigación para el diseño del currículo —es decir, objetivos educativos, métodos, materiales y evaluación- que permite a todas las personas desarrollar conocimientos, habilidades y motivación e implicación con el aprendizaje” (CAST, 
2015). Planteando currículos educativos flexibles y accesibles tal como lo propone el Diseño Universal para el Aprendizaje (DUA) puesto que las dificultades y barreras para el aprendizaje no son innatas, sino que nacen de la experiencia con herramientas y métodos inflexibles (Sala y Cols, 2014)

\section{Bases curricular}

Las Bases curriculares de acuerdo a la Ley General de Educación, $\mathrm{N}^{\circ} 20.370$, contempla un conjunto de objetivos de aprendizaje tales como conocimiento, habilidades y actitudes, las cuales se encuentran en total coherencia con los objetivos generales propuestos en esta Ley, utilizados en los niveles de educación parvularia, básica y media (Consejo Nacional de Educación [CNED], s/f, párr.1). Contemplan Objetivos de Aprendizaje (OA) por curso y asignatura, así como Objetivos de Aprendizaje Transversales (OAT) para el ciclo (CNED, s/f, párr. 2).

Es importante mencionar que actualmente se está viendo el uso de esta actualización curricular por parte de docentes, la cual por muchos no es completamente comprendida y da paso a que no se emplee de forma óptima, ya que esta debe ser la encargada de guiar la enseñanza y aprendizaje al complementar los planes y programas que el establecimiento opto por usar, incorporando los aspectos inclusivos del proceso de enseñanza aprendizaje.

De lo anterior, el enfoque de las bases curriculares es instaurar conocimientos, momentos de reflexión y contextualización como fruto de las actividades y experiencias alcanzables por todas y todos los estudiantes, por lo tanto es importante reflexionar sobre las metodología en la enseñanza para lograr un avance universal y accesible para todos. (Ministerio de Educación de Chile, 2015b).

\section{Modelos de procesamientos diversificados e inclusivos}

Existen estudios que apuntan a un modelo de canales de ingreso de la información. Este modelo, también llamado visual-auditivo-kinestésico² (V.A.K), toma en cuenta tres grandes vías de representación mental para la información, el visual, el auditivo y el kinestésico.

\footnotetext{
2 También se utilizan las palabras cenestésico o cinestésico para referirse al mismo concepto.
} 
La vía o sistema de representación visual permite ingresar imágenes abstractas (como números y letras) con una serie de posibilidades sobre lo escrito. El sistema de representación auditivo es el que permite oír voces, sonidos y música. El recuerdo de una melodía o conversación, o cuando se reconoce la voz del individuo que habla por teléfono, es cuando se utiliza el sistema de representación auditivo.

Lozano, 2006 citado en Alanís y Gutiérrez 2011, manifiesta que visualizar ayuda a establecer relaciones entre distintas ideas y conceptos, que en el caso del estudiante con un procesamientos más auditivo o kinestésico su desarrollo será mejor en la capacidad para abstraer y visualizar, lo que es debe entender como la individualidad de cada estudiante.

Por último, el sistema kinestésico permite distinguir sabores, olores, sentir el movimiento, por lo que se logra el uso de la corporalidad.

El modelo de procesamiento o canales de entrada V.A.K. su hipótesis y su compilación de diagnósticos, resulta interesante para los docentes, de modo que los atrae por sus grandes posibilidades de realización para alcanzar un aprendizaje más efectivo, en términos de reconocer las vías perceptuales ${ }^{3}$ por las que el estudiante prefiere la información y asimismo causa un tipo de aprendizaje, considerando las principales vías o conductos de acceso, que abarcan la visión, la audición y el movimiento (Rosabal, 2008).

Entre las ideas presentes en el curriculum educativo, es examinar al educando desde su singularidad y predilección para adquirir conocimientos, y la diversidad de estilos de aprendizaje existentes. La propuesta considera el contraste entre los seres humanos para aprender y que es trascendente para un pedagogo para entender el trabajo de la individualidad del estudiante, con el fin de integrar a sus prácticas pedagógicas inclusivas, lo que enriquecerá su práctica educativa. (Blanco, 2006).

Este modelo basado en los sistemas de representación toma en cuenta el criterio neurolingüístico, considerando la mejor vía para el ingreso de la información mediante los órganos sensoriales como: ojo, oído o el más extenso siendo el cuerpo. (Tapia, 2016)

\footnotetext{
${ }^{3}$ Perteneciente o relativo a la percepción mental o sensorial.
} 
López (2011) menciona: “Los sistemas de representación se desarrollan más, cuanto más los utilicemos. La persona acostumbrará a seleccionar un tipo de información y absorberá con mayor facilidad la información de ese tipo". Esto quiere decir, que mientras mayormente utilicemos los órganos de los sentidos, mayormente los potencializaremos, tal como López (2011, p.15) señala: "Utilizar más de un sistema implica que hay sistemas que se utilizan menos y, por lo tanto, que distintos sistemas de representación tendrán distinto grado de desarrollo”.

Este es un eje fundamental para la propuesta pedagógica inclusiva y diversificada, donde se pretende abarcar la mayor cantidad posible de estudiantes incluyendo como herramientas del docente los recursos didácticos que "son aquellas herramientas que el docente utiliza a diario sobre todo cuando induce a sus estudiantes a crear sus conocimientos a través de manipulación de materiales y ciertas actividades cotidianas" (Pola, 2015, p.98). Por lo tanto, se afirma que es un recurso que resulta útil o presenta un beneficio de manera directa o indirecta para el cumplimiento de un objetivo.

Ausubel (2010) menciona varias características para que los recursos sean novedosos, los cuales ayudan a una mejor planificación, favoreciendo la autonomía y estimulando la cooperación en grupos, estos son: recursos que sean fáciles de manipular, de carácter innovador por medio del desarrollo de ideas fomentando la creatividad, llamativos, capaces de captar la atención, avivando y reforzando las representaciones visuales, auditivas y kinestésicas, capaces de motivar y despertar el desarrollo de habilidades, destrezas y talentos innatos.

Haciendo mención el enfoques de enseñanza, se hace mención a que "no puede limitarse a un estilo de aprendizaje, ni mucho menos permanecer con un solo tipo de estrategias en el transcurso de tiempo del estudiante en la Unidad Educativa” (Gómez y López 2012, p. 13)

Caro (2008), analiza el Modelo de Estilos de Aprendizaje, que considera la capacidad del estudiante para ofrecer materiales pedagógicos dinámicos adaptados a cada estilo de procesamiento, entre ellos el Visual y el Auditivo.

A la hora de enseñar es indispensable saber que eje, o sistema de representación es el predominante, en una investigación de Alanís y Gutiérrez, sobre los estilos de aprendizaje se menciona la "predominancia en el estilo visual, considerando que los alumnos responden al 
contexto de la enseñanza, potenciando básicamente el ver, el cual está por encima de la escucha (auditivo), y ambos por sobre el estilo kinestésico.” (Alanís y Gutiérrez 2011, p.26)

Esto también es respaldado por las estadísticas que demuestran que "en las puntuaciones del Inventario de Estilos de Aprendizaje, la diferencia en la media se aproxima a privilegiar el canal visual, correspondiendo a este estilo de aprendizaje; 18.16, sobre el auditivo, 17.43 y sobre el kinestésico 15.35.” (Varela, 2006, p.11)

\section{Estilos cognitivos de Honey y Mumford, Estilos de aprendizaje}

Educarchile, realiza la siguiente interrogante: ¿Qué son los estilos de aprendizaje?, "se refiere al hecho de que se quiere aprender algo cada uno utiliza su propio método o conjunto de estrategias". (Educarchile, 2007, p. 1).

En los últimos años se han abordado diferentes teorías en el cual definen la forma de aprendizaje, más aún con el pasar de los años se observa el avance tecnológico y científico, y la influencia que conlleva a los estudiantes.

Es importante no utilizar los estilos de aprendizaje como una herramienta para clasificar a los alumnos en categorías cerradas. Nuestra manera de aprender evoluciona y cambia constantemente, como nosotros mismos" (Educarchile, 2007, p. 2).

El estilo de aprendizaje de cada persona, es según la escuela de programación neurolingüística (PNL) la manera preferida que cada uno tiene de captar, recordar, imaginar o enseñar un contenido determinado" (Educarchile, 2005, párr. 1).

En este contexto, lo principal son las experiencias de aprendizaje, es decir, que la enseñanza no sea simplemente que el profesor entregue información y que uno simplemente la reciba, sino que se empape de lo que se observa, siente con el olfato, percibir con el tacto, escuchar o percibir las cosas de manera general (Educarchile, 2005a, párr. 3).

Es importante que los alumnos mencionen al docente cual es el estilo de aprendizaje que más les acomoda (Educarchile, 2005), de esta manera, el profesor puede planificar de mejor forma sus clases que se ajusten a las capacidades intelectuales de cada niño y adolescente de una manera mucho más dinámica y efectiva. 
Por otra parte, no todas las personas aprenden de la misma manera que otros. Cada ser tiene una predisposición innata sobre asimilar y obtener conocimientos nuevos. Esto implica el estilo de cada persona y su manera de percibir el aprendizaje y que éste sea significativo.

Según Honey y Mumford los estilos de aprendizaje son cuatro: Activo, teórico, pragmático y reflexivo. (Piqueras, 2014).

Activos. Las personas activas son los que aprenden haciendo, es decir, deben interactuar netamente con el conocimiento cien por ciento y ser una experiencia de vida para lograr tal aprendizaje. (Piqueras, 2014, párr. 8).

Teóricos. Las personas teóricas son los que les gusta saber que hay detrás de todo lo que hablamos, es decir, deben estar implícitas las teorías. Los teóricos necesitan seguir a un modelo, concepto y estudios que sustenten lo que escuchan, de esta manera analizan y sintetizan la información que reciben para lograr una teoría lógica. (Piqueras, 2014, párr. 9).

Pragmáticos. Este tipo de personas necesitan llevar el aprendizaje a su diario vivir. Todos los conceptos tendrán una utilidad muy limitada a no ser que ofrezcan un amplio abanico de posibilidades de llevar a la práctica todo lo aprendido. Siempre están probando nuevas ideas, teorías y técnicas para corroborar si funcionan. Tener tiempo para pensar y hablar con otros sobre las conclusiones, casos reales, solución de problemas y debates suelen ser de mucha ayuda para los pragmáticos. (Piqueras, 2014, párr. 10).

Reflexivos. Este tipo de personas aprenden mediante la observación y reflexión de las cosas que ocurren, es decir, no es necesario que lo apliquen en su forma de vida, más bien realizan conclusiones válidas de las cosas que les sirvan. Los reflexivos aprenden mejor mediante los cuestionarios, observando actividades, entre otros. (Piqueras, 2014, párr. 11).

\section{Evaluación inclusiva}

Entre los postulados de la inclusividad en Chile, los autores Staimback, S. y Staimback, W (1999) mencionan que se debe incluir a todos los alumnos siempre y cuando los docentes realicen un esfuerzo para trabajar con los pares, adaptar el currículo, acoger a los alumnos y graduar las 
prácticas. Se debe tener en cuenta que la inclusión no se desarrolla con suavidad, por lo mismo se requiere que los adultos no se enfoquen en las cosas fáciles, sino más bien en buscar las posibles soluciones para lograr la inclusión socioeducativa satisfactoria.

De la idea anterior, se dice que "el aula de clases debe tomar consciencia y una postura anti-racista, anti-sexista, comprensiva e igualadora y trabajar con los alumnos y alumnos en cómo combatir las desigualdades sociales y crear una ciudadanía comprometida con la inclusión”. (Ministerio de Educación de España [MECD], 2012, p.2)

Nombrar una sala de clases "inclusiva" no quiere decir que realmente lo sea. No se trata de un simple nombre, sino de las cosas que ocurren día a día con los estudiantes. Los jóvenes deben sentir que son bienvenidos y colaborativos con sus pares, que se forme un ambiente de confianza y tengan el respeto cuando se requiera con el fin de lograr una enseñanza aprendizaje y tener mejores oportunidades de mejora. $\left(\mathrm{MECD}^{4}, 2012\right.$, p.6)

Para incrementar la inclusión en las aulas, el establecimiento debe constituir equipos de aula por cada curso que cuente con estudiantes con NEE.

La idea del Equipo de Aula a un grupo de profesionales que trabajan colaborativamente en el espacio del aula, es una estrategia que cumple con la finalidad común de mejorar la calidad de la enseñanza y de los aprendizajes, en un marco de valorización de la diversidad y de respeto por las diferencias individuales de los estudiantes. Este equipo está conformado por los profesores de aula, profesores especialistas y profesionales asistentes de la educación y dependiendo de la realidad social pueden incorporarse intérpretes de lengua de señas chilena. El Equipo de Aula se desempeñará en la sala de clases y realizará reuniones de planificación y otras acciones de apoyo a los estudiantes, a las familias y a los docentes, fuera del aula regular. (Ministerio de Educación de Chile 2013, p. 40)

Los Equipos de Aula funcionan son una estrategia inclusiva del sistema escolar en un Programa de Integración Escolar, regido por la Ley 20.201, cuyo objetivo principal es entregar apoyos a los estudiantes que presentan Necesidades Educativas Especiales (NEE), ya sea

\footnotetext{
${ }^{4}$ Ministerio de Educación, Cultura y Deporte, España.
} 
transitorias o permanentes, con el fin de equiparar oportunidades para su participación y progreso en los aprendizajes del curriculum nacional. (MINEDUC, 2013, p. 5)

\section{Estilos de enseñanza}

En los últimos años se han desarrollado diferentes formas de aprendizaje, centrados en el alumno, donde el docente considere la diversidad en el aula con los estilos de aprendizaje de cada niño y joven, y de esta forma aplicar un enseñanza significativa para todos los estudiantes.

En Chile los establecimientos educacionales trabajan con diferentes métodos, según Educarchile (2011) estos métodos serían:

- Método Montessori: Que se define como una filosofía de vida más que un sistema de aprendizaje. Su principio básico es "seguir al niño". El maestro hace el programa de trabajo, pero es el niño el que dará los tiempos y la pauta del aprendizaje.

- Método Freinet: Rige los intereses del alumno, ya que son el motor de la vida diaria de la escuela, que se organiza en torno a un tema propuesto por los niños. Los trabajos se exponen y los compañeros deben opinar. La expresión oral y plástica es básica en este método. El Colegio Rubén Darío de Santiago, entre otros, trabaja en Chile el Método Freinet.

- Método Waldorf; Se practica en Chile desde hace más de una década. Este enfoque plantea que pensamiento, sentimiento y voluntad tienen igual importancia en el desarrollo de un niño sano, es por ello, que el currículum contempla estimular el desarrollo de las capacidades cognitivas, morales, artísticas, manuales, musicales y corporales considerando la etapa de desarrollo del niño.

Existe un instrumento en Chile llamado el Marco para la Buena Enseñanza ${ }^{5}$ que dicta lo siguiente:

Supone que los profesionales que se desempeñan en las aulas, antes que nada, son educadores comprometidos con la formación de sus estudiantes. Supone que para lograr la buena enseñanza, los docentes se involucran como personas en la tarea, con todas sus

\footnotetext{
${ }^{5}$ Ha sido elaborado por el Ministerio de Educación, a partir de la reflexión tripartita de los equipos técnicos de éste, de la Asociación Chilena de Municipalidades y del Colegio de Profesores, y teniendo a la vista la experiencia nacional e internacional sobre criterios acerca del desempeño profesional de docentes de los sistemas escolares.
} 
capacidades y sus valores. De otra manera, no lograrían la interrelación empática con sus alumnos, que hace insustituible la tarea docente.

Existen cuatro dominios del marco que determina alguna actitud de la enseñanza:

1) Preparación de la Enseñanza.

2) Creación de un ambiente propicio para el aprendizaje.

3) Enseñanza para el aprendizaje de todos los estudiantes.

4) Responsabilidades profesionales.

Por lo señalado, es que la preocupación incluye una diversidad en las buenas prácticas educativa que es un aporte para el proceso de la enseñanza.

\section{Aprendizaje significativo}

Educarchile (2007) describe que durante el aprendizaje es importante conocer la estructura cognitiva del alumno. En esta sentido, cita al autor Ausubel (1983) el que plantea:

El aprendizaje del alumno depende de la estructura cognitiva previa que se relaciona con la nueva información, debe entenderse por "estructura cognitiva", al conjunto de conceptos, ideas que un individuo posee en un determinado campo del conocimiento, así como su organización. (pág. 5)

El aprendizaje significativo de un estudiante no solo es la conexión de lo que ya sabe y el nuevo conocimiento. Ausubel (1983) citado por Educarchile (2007, p. 5) "distingue tres tipos de aprendizaje significativo". El primero, Aprendizaje de Representaciones, consiste en la atribución de significados a determinados símbolos; el segundo, Aprendizaje de Concepto, son los "objetos, eventos, situaciones o propiedades de que posee atributos de criterios comunes y que se designan mediante algún símbolo o signos" (Ausubel 1983:61); y el tercero, Aprendizaje de proposiciones, en el cual exige captar el significado de las ideas expresadas en forma de proposiciones.

Ausubel, Novak y Hanesian (1978) citado por Educarchile (2007, p. 17), explican que "la esencia del aprendizaje significativo reside en el hecho de que las ideas están relacionadas simbólicamente y de manera no arbitraria (no al pie de la letra) con lo que el alumnado ya sabe". 


\section{Inteligencias múltiples}

En este mundo globalizado donde las tecnologías han avanzado y han permitido obtener mayor alcance de información, se han encontrado distintas teorías que tratan de explicar las diversas inteligencias que el ser humano posee.

Cuando sale a flote la interrogante de cómo es el aprendizaje de cada individuo y cómo es posible lograr potenciar esa habilidad. Es cuando se presenta la disyuntiva de que, si todos aprenden de la misma forma o no, o si son capaces de trabajar de la misma forma o hay algunos que son mejores en ciertas áreas.

La teoría de las inteligencias múltiples según Llor y Cols (2012) menciona que se logra reconocer las diferentes formas de enseñar y aprender además de proporcionar procedimientos dinámicos de la evaluación cognitiva.

Varias son las maneras de ver y definir la inteligencia ya que al ser un fenómeno de carácter complejo por la dependencia en el enfoque disciplinario del que la utilice esta influye en su variación. A partir de esto, "las definiciones de inteligencia se pueden organizar en varios grupos como las psicológicas, las biológicas y las operativas” (Suárez, Maiz y Meza, 2010, párr. 9)

Son varios los estudios que se han realizado a partir de lo que postula Howard Gardner con las inteligencias múltiples, uno de estos investigadores es Chan (2001) citado en Llor y Cols (2012), el cual tuvo como objetivo estudiar la viabilidad de la utilización de los auto informes de las inteligencias múltiples en la identificación de diferentes aspectos de la súper dotación .

También realizó otro estudio el cual se encuentra dentro de los parámetros que son relevantes dentro de esta investigación donde Chan (2004) citado en Llor y Cols (2012), midió los perfiles de las ocho inteligencias de 133 alumnos utilizando la valoración de sus madres, padres, maestros compañeros y autoevaluación, con el propósito de entender mejor el potencial y las necesidades de estos alumnos y también explorar si se podía predecir la creatividad y el liderazgo partiendo de los perfiles de las Inteligencias múltiples.

Suárez et al, (2010), concibe la idea en su análisis sobre inteligencias múltiples de manera facilitadoras, manifiesta: 
La utilización de la teoría de inteligencias múltiples permite y facilita la aplicación de novedosas estrategias que resultan motivantes, integradoras y creativas a la hora de que los estudiantes pongan en práctica su rol protagónico, donde la forma de manejar el conocimiento les permita obtener una visión de la realidad y logre superar los límites del saber habitual, permitiendo un desarrollo en su potencial creativo y la activación de otras inteligencias (párr. $15)$.

\section{Propuestas inclusivas de la educación a nivel curricular}

De acuerdo al contexto expuesto anteriormente, según la situación de la enseñanza en Chile con sus diversos autores y las bases curriculares en conjunto con las modificaciones y cambios que se han efectuado, de igual manera es de suma importancia conocer las propuestas inclusivas en la educación chilena en conjunto a toda la diversidad que estas nos presentan en sus leyes, abarcando como pilar el $\mathrm{DUA}^{6}$ para finalmente llegar a la planificación diversificada.

\section{Diseño Universal de Aprendizaje}

El Diseño Universal de Aprendizaje (DUA) como declara el $\mathrm{CAST}^{7}$ es un marco que confronta a los currículos inflexibles, que hoy en día es la barrera principal para promover la enseñanza, ya que generan obstáculos involuntarios que dificultan el acceso del aprendizaje debido a que no alcanza al $100 \%$ de estudiantes, como lo son los alumnos superdotados o los discapacitados, y esta vulnerabilidad se refleja en un currículum pobre puesto que pasa por alto y no atiende muchas de estas necesidades (CAST, 2011).

El decreto 83, hablando sobre el DUA, dice que "es una estrategia de respuesta a la diversidad, cuyo fin es maximizar las oportunidades de aprendizaje de todos los estudiantes, considerando la amplia gama de habilidades, estilos de aprendizaje y preferencias" (Ministerio de Educación de Chile, 2015a, p.19).

Tipos de planificación

Al momento de explicar que es una planificación diversificada es importante conocer si existen otros tipos de planificación o de como la educación en Chile está enfrentando la organización de las clases.

\footnotetext{
${ }^{6}$ Diseño Universal de Aprendizaje.

${ }^{7}$ Center for Applied Special Technology (Centro de Tecnología Especial Aplicada).
} 
En Chile el Ministerio de Educación, expresa que la planificación tiene como objetivo el ordenar y definir los tiempos en concordancia con lo que propone el currículum nacional en relación a los aprendizajes que son abarcados durante el año escolar (Ministerio de Educación de Chile [MINEDUC], 2007), la planificación puede verse según dos clasificaciones así lo menciona Educarchile, (2005a) las cuales son en cuanto al tiempo invertido o modelo pedagógico.

Una planificación de acuerdo al tiempo invertido se desglosa en tres intervalos de tiempo, una planificación de tipo anual donde se trata de plasmar o diseñar los aprendizajes esperados durante todo el año, la cual se compone de varias unidades didácticas y todas estas en completa coherencia, también se organiza de forma más acotada a través de unidades didácticas la cual no se rige por tiempos sino más bien es utilizada por el docente asignándole las horas pedagógicas necesarias para alcanzar la adquisición de los aprendizajes, y por último la planificación clase a clase la cual el docente plantea el orden y actividades que se realizara durante una clase, esto es muy variable ya que por razones de la diversidad en el aula muchas veces se debe ir moldeando esta planificación, por lo que ya no se exige obligatoriamente al docente, más bien pueda ser de ayuda y guía en su desempeño (Educar chile,2005b).

En Chile los establecimientos educacionales trabajan con las planificaciones según el enfoque de intervalo de tiempo invertido, según Currículum en Línea (2011) más detallada cada una de estas formas podemos observar en la siguiente tabla.

El otro tipo de planificación es aquella que demanda una estructura a nivel pedagógica, la cual tiene como fin asegurar la cobertura curricular y prever las necesidades que emergerán durante el proceso de enseñanza y aprendizaje (MINEDUC, 2007).

Según Flórez en Educarchile (2005b) estas serían:

Planificación en T: Es un tipo de planificación donde se estructuran en cuatro secciones que son capacidades - destrezas, valores - actitudes, procedimientos- estrategias y contenidos conceptuales. Está inserta tanto en el modelo cognitivo ${ }^{8}$ como en el constructivista $^{9}$. Esta planificación es un buen modelo para ser usada como planificación

\footnotetext{
${ }^{8}$ Habilidades adquiridas.

${ }^{9}$ Forma de adquirir las habilidades.
} 
anual pues es de carácter amplio, ya que permite abordar todos los aspectos importantes, pero posee una desventaja la cual es que carece de evaluación.

Planificación heurística: Asocia como punto principal al modelo cognitivo y puede ser muy útil para el desempeño y organización del docente, ya que logra evidenciar teóricamente lo que está detrás de su unidad didáctica. Se debe escoger una pregunta central, la cual será respondida de forma conceptual a un lado, por el otro lado deberá ir la metodología que permitirá desarrollar los conceptos. Es un modelo complejo para usar, pues no siempre es fácil diferenciar las distintas categorías.

Planificación en trayecto: Es un tipo de planificación que sirve para elaborar unidades didácticas y no planificaciones anuales, contempla cuatro casilleros principales los cuales son el aprendizaje esperado, contenidos, actividad y evaluación todo esto inserto en el modelo cognitivo y constructivista.

Una ventaja es que se trabaja con la nomenclatura del actual marco curricular e incorpora los elementos necesarios para una buena planificación como lo es el que, el para qué y el cómo.

\section{Conclusión}

Hoy en día es importante ver como actualmente existe un desafío en la educación actual para avanzar en la integración de todos los estudiantes. La enseñanza -aprendizaje pueda ser más significativa en el aula de clases, donde el punto importante es la diversidad que existe a la hora de aprender y es por eso que los contextos inclusivos utilizan una planificación diversificada, puesto que está pensada y fundamentada en el diseño universal de aprendizaje que considera siempre la preocupación de que cada estudiante pueda aprender y que no sea limitado por qué no se potenciaron sus fortalezas o simplemente no se le prestó atención.

La información sobre las metodologías que se están empleando pretendió contribuir con ideas para los conocimientos que resulten ser significativos. 
El camino de la innovación muchas veces no es fácil, ya que serán muchas las limitantes que pueden ir apareciendo en el transitar, lo que da implica una propuesta de oportunidades en la búsqueda del mejoramiento de la educación. 


\section{Referencias}

Alanís, P., y Gutiérrez, D. (2011), Los estilos de aprendizaje en estudiantes de Telesecundaria. Recuperado de https://dialnet.unirioja.es/descarga/articulo/4034711.pdf

Álvarez, Z., Porta, L., y Sarasa, M. (2011). Buenas prácticas docentes en la formación del profesorado: relatos y modelos entramados. Revista de Currículum y Formación de Profesorado ISSN: 1138-414. Recuperado de www.redalyc.org/pdf/567/56717469016.pdf

Ausubel, D. (2010) Psicología educativa: un punto de vista cognoscitivo. México: Trillas

Blanco, R. (2006). La equidad y la inclusión social: uno de los desafíos de la educación y la escuela hoy. Revista Electrónica Iberoamericana sobre Calidad, Eficacia y Cambio en Educación, 4(3). Recuperado de : www.redalyc.org/pdf/551/55140302.pdf

Caro, E., y Monroy, M. (2008). Relación de los Ambientes Hipertextuales de Aprendizaje Gráfico y Sonoro, con los Estilos de Aprendizaje Verbal y Visual. Revista Avances en Sistemas e Informática, 5(2).

Center for Applied Special Technology (CAST) (2011). Universal Design for learning guidelines version 2.0. Wakefield, MA: Author. Traducción al español versión 2.0 (2013): Alba Pastor, C., Sánchez Hípola, P., Sánchez Serrano, J. M. y Zubillaga del Río, A. Pautas sobre el Diseño Universal para el Aprendizaje (DUA).Texto completo (versión 2.0).

Consejo Nacional de Educación [CNED] (s/f), Educación Escolar, Marco Curricular y Bases $\begin{array}{llll}\text { curriculares de } & \text { Chile. } & \text { Recuperado }\end{array}$ http://www.cned.cl/public/Secciones/SeccionEducacionEscolar/marco_curricular.aspx

Cox, C. (2001) El currículum escolar del futuro. Unidad de Currículum y Evaluación, Ministerio de Educación, 4(2), 213-232.

Cox, C. (2011) Currículo escolar de Chile: génesis, implementación y desarrollo. CEPPE-Facultad de Educación PUC. Revue International de Education de Sevres, 56.

Educarchile. (2005a). Calidad y diversidad en la educación chilena. Santiago, Chile. Recuperado de http://www.educarchile.cl/ech/pro/app/detalle?ID=91530eks201516169102 
Educarchile. (2005b). Modelos pedagógicos y planificación: un poco de historia Recuperado de http://www.educarchile.cl/ech/pro/app/detalle?id=78295.

Educarchile (2011). Los métodos que hacen la diferencia. Santiago, Chile. Recuperado de http://www.educarchile.cl/ech/pro/app/detalle?id=73999.

Flórez, T. (2005). Tipos de planificación. Recuperado de http://www.educarchile.cl/ech/pro/app/detalle?id=78294.

Freire, P. (1972). Pedagogía del oprimido. Argentina: Editorial Siglo XXI.

Fuentes, R., Gamboa, J., Morales, K., Retamal, N., y San Martín, V. (2012) Jean Piaget, aportes a la educación del desarrollo del juicio moral para el siglo XXI. Revista Convergencia Educativa. Recuperado de https://www.academia.edu/9740093/JEAN_PIAGET_APORTES_A_LA_EDUCACI\%C3\%9 3N_DEL_DESARROLLO_DEL_JUICIO_MORAL_PARA_EL_SIGLO_XXI

Gómez, A., y López, H. (2012). Estilos de aprendizaje en los estudiantes universitarios con base en el modelo de hemisferios cerebrales. Revista Académica de Investigación TLATEMOANI. España. Recuperado de http://www.eumed.net/rev/tlatemoani/11/hemisferioscerebrales.pdf

Guerra, C., Vargas, J., Castro, L., Plaza, H., y Barrera, P. (2012). Percepción del Clima Escolar en Estudiantes de Enseñanza Media de Valparaíso de Colegios Municipales, Particulares Subvencionados y Particulares. Revista Estudios Pedagógicos 38(2), 103-115, 2012. Recuperado de : http://www.scielo.cl/pdf/estped/v38n2/art07.pdf

Ministerio de Educación de Chile. (2007). Ley de Educación, sobre subvenciones a establecimientos educacionales $y$ otros cuerpos legales. Recuperado de: https://www.leychile.cl/Navegar?idNorma=263059

Ministerio de Educación de Chile. (2009). Ley General de Educación, Chile. Recuperado de http://www.leychile.cl/Navegar?idNorma=1006043\&idParte $=$.

Llor, L., Prieto, F., García, F., Hernández, D., Sáinz, M., Sánchez, P. y Fernández, C. (2012). Inteligencias múltiples y alta habilidad. Aula abierta,40(1), 27-38. Recuperado de https://dialnet.unirioja.es/servlet/articulo? codigo=3791837. 
López, R. (2011) Modelo de Aprendizaje VAK. Recuperado el domingo 4 de Octubre de 2016. Recuperado de http://generandoconocimiento.blogspot.com/2011/02/modelo-deaprendizajevak.html

Machado, A. (2005). Calidad y diversidad en la educación chilena. Santiago de Chile.

Ministerio de Educación de Chile. (2016). Orientaciones técnicas: La planificación como un proceso sistémico y flexibl., Santiago, Chile.

Ministerio de Educación de Chile. (2015). Orientaciones para planificar el aprendizaje. Recuperado de http://www.curriculumenlineamineduc.cl/605/w3-article-14601.html

Ministerio de Educación de Chile. (2015a). Decreto 83 "Diversificación de la enseñanza", Santiago, Chile.

Ministerio de Educación de Chile. (2015b). Bases Curriculares. Santiago, Chile.

Ministerio de Educación de Guatemala (2006). Herramientas de evaluación en el aula. Guatemala / USAID.

Ministerio de Educación de España. (2012). Educación Inclusiva. Iguales en la diversidad.

Ministerio de Educación de Chile. (2012). Orientaciones técnicas para Programas de Integración Escolar (PIE). Santiago chile.

Ministerio de Educación de Chile. (2013). Orientaciones para el trabajo colaborativo y evaluación de un pie, División de Educación General, Unidad de educación especial. Recuperado de http://portales.mineduc.cl/usuarios/edu.especial/doc/201310081640100.orientacionesPIE2013 .pdf

Piqueras, C. (2014). Los cuatro estilos de aprendizaje de Honey y Mumford. [Entrada de blog]. Recuperado de https:/www.cesarpiqueras.com/los-cuatro-estilos-de-aprendizaje-de-honey-ymumford/

Pola, F. (2015). El uso de materiales didácticos favorece el aprendizaje significativo de los alumnos. Recuperado el sábado 13 de Febrero de 2016. Disponible en: http://www.eumed.net/libros-gratis/2015/1457/constructivismo.htm

Posner, G. (1998) Análisis del curriculum. Colombia: McGraw-Hill/Interamericana 
Rodríguez, D. (2008). Buenas prácticas en el ámbito educativo y su orientación a la gestión del conocimiento. Revista Educación, 17(32). Recuperado de: http://revistas.pucp.edu.pe/index.php/educacion/article/view/1537.

Rosabal, G. (2008) Estilos de aprendizaje y educación instrumental. Revista La Retreta, 2. Recuperado de: http://laretreta.net/0102/articulos/estilosdeaprendizaje.html

Sala, I., Sánchez, S., Giné, C., y Díez, E. (2014). Análisis de los distintos enfoques del paradigma del diseño universal aplicado a la educación. Revista Latinoamericana de Educación Inclusiva. 8(1). Recuperado de http://www.rinace.net/rlei/numeros/vol8-num1/art9_htm.html

Staimback, S., y Staimback, W. (1999) Aulas inclusivas. Madrid: Narcea.

Suárez, J., Meza, F y Meza, M. (2010). Inteligencias múltiples: Una innovación pedagógica para potenciar el proceso enseñanza aprendizaje. Investigación y Postgrado, 25(1), 81-94. Recuperado de http://www.scielo.org.ve/scielo.php?script=sci_arttext\&pid=S131600872010000100005

Tapia, L. (2016). Los recursos didácticos del área de lengua y literatura y los procesos psíquicos de la atención en los y las estudiantes de décimo año de e.g.b. De la unidad educativa general eloy alfaro, de la ciudad de ambato (Tesis de pregado). Universidad Técnica De Ambato, Ecuador.

Varela, M. (2006). Estilos de Aprendizaje. Departamento de Biología Celular y Tisular, Facultad de Medicina, UNAM. Recuperado de http://bq.unam.mx/wikidep/uploads/MensajeBioquimico/Mensaje_Bioq06v30p1_11_Margari ta_Varela.pdf 